\title{
La resiliencia, camino a la reconstrucción social en contextos de conflicto: una experiencia de educación religiosa en perspectiva decolonial
}

David Emmanuel Vides SAN JuAN ${ }^{1}$

Resumen: Partiendo del contexto colombiano, caracterizado por los procesos de paz y el denominado posconflicto luego de medio siglo de lucha armada, se presentan reflexiones suscitadas por una experiencia pedagógica, desde el área de Educación Religiosa y en perspectiva interdisciplinar con las Ciencias Sociales, encaminada a la reconstrucción del tejido social mediante el fortalecimiento de la propia identidad y la comprensión del otro, que permita afianzar un desempeño político desde la transformación social. La presentación se da a partir de las cuatro claves de comprensión de las pedagogías descoloniales; así: la descolonización del saber, que plantea la íntima conexión entre los ámbitos político y religioso, y sus implicaciones en el área de Educación Religiosa; la descolonización del ser, donde a partir del reconocimiento de la trascendencia como dimensión humana fundamental se derivan algunas consideraciones en lo político; la descolonización de las prácticas culturales, donde se asume el hecho religioso como hecho cultural lo que constituye una puerta de entrada a la reflexión existencial; finalmente, la descolonización del currículo, que plantea la Resiliencia como referente generador de sentido, a partir de los ejes de Paz, Reconciliación y Memoria.

Palabras clave: Resiliencia. Memoria histórica. Educación religiosa. Trascendencia. Pedagogías decoloniales.

\section{A resiliência, o caminho para a reconstrução social em contextos de conflito: uma experiência de educação religiosa em uma perspectiva decolonial}

Resumo: A partir do contexto colombiano, caracterizado por processos de paz e pelo denominado pós-conflito após meio século de luta armada, são apresentadas reflexões decorrentes de uma experiência educacional com base na área da educação religiosa, em uma perspectiva interdisciplinar com as Ciências Sociais, visando à reconstrução do tecido social por meio do fortalecimento da própria identidade e do entendimento do outro, o que permite consolidar uma atuação política a partir da transformação social. A apresentação é dada a partir das quatro chaves de compreensão das pedagogias decoloniais: a descolonização do conhecimento, que levanta a conexão íntima entre as esferas política e religiosa, e suas implicações na área da Educação Religiosa; a descolonização 
do ser, em que do reconhecimento da transcendência como dimensão humana fundamental derivam algumas considerações políticas; a descolonização das práticas culturais, em que o fato religioso é assumido como um fato cultural que constitui um portal para a reflexão existencial; por fim, a descolonização do currículo, que propõe a resiliência como um gerador de sentido, com base nos eixos da paz, reconciliação e memória. Palavras-chave: Resiliência. Memória histórica. Educação religiosa. Transcendência Pedagogias decoloniais.

\title{
The resilience, the way to social reconstruction in contexts of conflict: an expe- rience of religious education in a decolonial perspective
}

\begin{abstract}
From the Colombian context, characterized by the processes of peace and the so-called post-conflict after half a century of armed conflict, reflections arising from a pedagogical experience are presented, from the area of Religious Education and in interdisciplinary perspective with the Social Sciences, aimed at reconstruction of the social fabric through the strengthening of the identity and the understanding of the other, which allows to consolidate a political performance from the social transformation. The presentation arises from the four keys of understanding of decolonial pedagogies; thus: the decolonization of knowledge, which raises the intimate connection between the political and religious spheres, and its implications in the area of Religious Education; the decolonization of being, where from the recognition of transcendence as a fundamental human dimension some political considerations derive; the decolonization of cultural practices, where the religious fact is assumed as a cultural fact that constitutes a doorway to existential reflection; finally, the decolonization of the curriculum, which proposes Resilience as a generator of meaning, based on the axes of Peace, Reconciliation and Memory.

Keywords: Resilience. Historical memory. Religious education. Transcendence. Decolonial pedagogies.
\end{abstract}

\section{Introducción: dos presupuestos fundamentales}

La coyuntura actual de la sociedad colombiana, marcada por la reciente firma de los acuerdos de paz y el difícil proceso de implementación, exige la apertura de perspectivas que permitan canalizar en el campo educativo los elementos necesarios para una auténtica reconstrucción del tejido social, especialmente en un escenario donde no hay claridad plena en la finalización del conflicto armado y urge consolidar las garantías de no-repetición. En ese contexto, la consideración de un nuevo tipo de ciudadanía pasa por el reconocimiento del otro y su inclusión en la constitución de un proyecto nacional, y supone una revisión de posibilidades y alternativas desde la escuela.

La pretensión fundamental es el planteamiento de algunas reflexiones surgidas de una experiencia pedagógica de Educación Básica y Media, en el Colegio Distrital Bravo Páez, en la ciudad de Bogotá; desde el área de Educación Religiosa y en perspectiva interdisciplinar con las Ciencias Sociales, se han abordado 
prácticas de memoria histórica en los espacios escolares, con el fin de fomentar una comprensión integral de las circunstancias coyunturales del país, ligada al fortalecimiento de la propia identidad y a la comprensión del otro, que permita afianzar un desempeño político desde la transformación social.

Un presupuesto inicial, es el planteamiento de la violencia política en el caso colombiano como un fenómeno cuyas raíces se hunden en la ausencia de reconocimiento del otro, evidenciada en un complejo entramado de situaciones que pasan por el abandono estatal, la desigualdad social, los vicios del aparato político institucional, y el fraccionamiento del país en regiones que no constituyeron de forma clara un proyecto nacional (CELIS, 2009). Se estableció así un auto-reconocimiento de la exclusión que coadyuvó el surgimiento y crecimiento de movimientos de reivindicación con pretensiones de raigambre política; en ese momento, la apertura a su inclusión, brindando garantías y oportunidades de participación, podría haberse constituido en una oportunidad de reorganización y reconocimiento de las necesidades de ciertas comunidades; sin embargo, lo que terminó por consolidarse fue la lógica de la guerra con su noción polarizada amigo-enemigo.

En ese sentido, es posible explicar la violencia como síntoma de las dinámicas de exclusión política y en ello, la ausencia de un reconocimiento del otro-campesino, y su invisibilización. Frente a ello, se requiere de forma apremiante una reflexión en torno a la ciudadanía y las condiciones de empoderamiento de la sociedad civil, así como, en torno a la necesidad de construcción conjunta a partir de lo diverso; en otras palabras, el reto de reconstruir el tejido social, donde la educación y en particular, la formación política tiene un papel fundamental.

Dicho esto, la invisibilización y negación de la diferencia, evidenciada en dinámicas de exclusión y marcada polarización, constituyen una perspectiva de análisis de la situación política, y así mismo un paradigma posibilitador de respuestas en el campo educativo. Ahora, la importancia de la situación social y política, como momento histórico coyuntural en el país, convierte los ejes de fortalecimiento del ser personal y reconocimiento del papel individual y comunitario, en exigencias apremiantes, a partir de una labor de renovación pedagógica en los espacios más llanos del proceso; una aproximación a las pedagogías decoloniales como "apuesta integradora de constitución de subjetividades éticas" (ARGÜELLO PARRA, 2015 , p. 28), constituye un segundo presupuesto para la experiencia pedagógica.

Partiendo de la ampliación del concepto colonialidad, -que supera la comprensión tradicional de un periodo histórico específico para identificarlo como una forma de relación entre el centro y la periferia vigente en nuestros contextos actuales-, se asume una perspectiva de colonialidad global que denuncia las diferentes exclusiones generadas por el proyecto moderno y más aún, expone que el proceso de descolonización es una tarea aún pendiente. Las implicaciones de esta perspectiva van desde el reconocimiento de las distintas interconexiones existentes entre las estructuras que constituyen el engranaje social, hasta la 
crítica a la modernidad hegemónica basada la supremacía de la razón centrada en el sujeto, pasando por la conformación de un programa decolonial con tres características fundamentales: diacrítico, al constituirse en un ejercicio reflexivo que involucra también la propia postura, dialógico, al promover un ejercicio de intercambio y enriquecimiento mutuo entre los diferentes actores, y oposicional, al tener como referente permanente el opuesto con el fin de señalar como eje primordial la posibilidad de la diferencia (ARGÜELLO PARRA, 2015).

Dicho esto, el proyecto decolonial aboga por la superación de la comprensión de la diferencia en términos de inferioridad con respecto a quien clasifica desde un paradigma único y universal; nos enfrentamos nuevamente al tema de la negación del otro, del diferente. Por su parte, la educación no es ajena a esta racionalidad monotópica moderna, lo cual puede evidenciarse en las manifestaciones y estructuras emergentes que siguen la lógica de la economía, llevando a una mercantilización de la enseñanza, reduccionista de la pluridimensionalidad del ser humano. En oposición a ello, la pedagogía decolonial plantea derroteros para permitir a lo otro y al otro ser; y aunque no existen fórmulas establecidas para ello, siendo la reflexión profundamente contextual, pueden plantearse algunas claves de comprensión que reconocen la pedagogía como una tarea social, una posibilidad de crítica y autocrítica y una oportunidad de transformar realidades. Siguiendo a Mignolo, Argüello Parra (2015) sintetiza estas claves en cuatro: primero, la decolonización del saber, relacionada con las formas de validación y producción del conocimiento, insistiendo en el intercambio entre los conocimientos legitimados por la ciencia y aquellos emergentes de cara a las necesidades de las comunidades, en atención a la cotidianidad y sus problemas; segundo, la decolonización del ser, que traduce la clave anterior a la construcción de subjetividades, creando nuevos estilos de vida y de relacionarse; tercero, la decolonización de prácticas socioculturales, ligadas a la etnia, la clase o el género, reivindicando los diversos modos de ser otro a nivel individual y colectivo, en una coexistencia, que evite el aislamiento y la constitución de guetos; cuarto, la decolonización del currículo, ya en el ámbito escolar formal, como apertura a la circulación de los saberes emergentes y de las comprensiones culturales distintas (ARGÜELLO PARRA, 2015).

Esta apuesta por hacer visible al otro en la escuela, supone integrar en su cotidianidad los debates discusiones y problemáticas que expresan la complejidad del mundo en su diversidad. Para el caso del abordaje del conflicto armado en Colombia, y habiendo presentado una perspectiva de la violencia en Colombia como síntoma de un problema de alteridad, en términos de invisibilización y negación del otro, se plantea para el desarrollo del artículo, la ubicación de la experiencia en el marco reflexivo de las pedagogías decoloniales, siguiendo las cuatro claves de comprensión expuestas. Siguiendo la línea de reflexión de una Educación Religiosa unida a la formación política, se plantean los elementos de decolonización que es posible evidenciar en la experiencia pedagógica. 


\section{Decolonización del saber: co-implicación de lo político y lo religioso}

Respecto a la forma de validación del conocimiento, el esfuerzo se encamina a hacer evidente lo que en un escenario educativo no es tenido en cuenta tan fácilmente, y que puede llegar a contravenir el discurso hegemónico. Para el caso de la experiencia pedagógica que se presenta, adquiere relevancia, por una parte, el replanteamiento de las relaciones entre lo religioso y lo político que hoy en día superan la separación tradicional entre lo privado y lo público, y por otra, las implicaciones de cara a la consideración del espacio pedagógico de la Educación Religiosa como una posibilidad de formación política.

En primer lugar, siguiendo a Duch (2014), las relaciones entre lo religioso y lo político han marcado profundamente la historia de los pueblos, de forma que las estructuras políticas de la Religión interactúan con las estructuras religiosas de la Política, produciéndose unas sinergias de significación entre los términos políticos aplicados a la Religión, así como algunas particulares dinámicas religiosas que entran en juego en el quehacer político. Frente a esto pierde crédito la idea moderna de separación de lo religioso y lo político en dos esferas distintas; mientras la escisión parece clara en la teoría, en la práctica ambas esferas están íntimamente ligadas. La comprensión de este postulado pasa por la distinción entre lo religioso y las religiones, así como entre lo político y las políticas; en este sentido, las religiones históricas no serían sino articulaciones culturales de lo religioso. De esta manera, teóricamente lo político aparece como referido al ámbito administrativo de las relaciones entre los seres humanos mientras que lo religioso vendría a ser el ámbito de la interioridad de la experiencia y las relaciones con un poder superior y trascendente; la realidad hace que esta separación teórica no sea correspondiente con lo que sucede a nivel práctico, más aún, teniendo en cuenta las implicaciones de lo religioso en temas concretos que afectan la vida política.

En esa línea, las respuestas que dan las personas concretas a situaciones particulares son distintas de acuerdo con aquello a lo que se sientan ligadas de forma incondicional. En otras palabras, las convicciones religiosas que se arraigan en la dimensión trascendente o en la vida interior terminan influyendo de forma directa en las consideraciones y opciones políticas porque ambas están co-implicadas; de hecho, las convicciones dejan de ser únicamente religiosas o únicamente políticas, superando la tendencia moderna de obtener ideas claras y distintas relacionadas con cada uno de los ámbitos de lo humano. La novedad que se plantea es que más allá de la presencia de lo religioso y lo político en forma de una yuxtaposición permanente, lo que acontece es una co-implicación de ambos componentes. Así, los fenómenos religiosos como concreciones limitadas y determinadas culturalmente de lo religioso, van más allá de la vida espiritual de los fieles intentando superarla para dominar la vida pública de acuerdo a intereses particulares. En correspondencia lo político que tampoco es claro sino 
a través de mediaciones políticas concretas, no sólo se limita a la administración de lo público, sino que influye sobre la conciencia los individuos en aspectos que en buena parte tienen influencia religiosa.

En este sentido, problemas políticos contemporáneos, encuentran en lo político-religioso una forma alternativa de comprensión del mundo, que permite fundamentar juicios y decisiones; ejemplo claro de ello, es la ruptura de la confianza en las instituciones, que se constituye en una dinámica vital cuya crisis amerita una solución que supere el simple acuerdo político, esto es, debe manifestarse desde la sanación de dimensiones más profundas del ser humano. Así, temas como el restablecimiento de la confianza, la importancia de la tierra, las relaciones de poder, la organización de los estados, entre otros, resultan vinculados íntimamente, co-implicados a aquello que nos atañe de forma incondicional.

Con base en lo anterior, se hace necesario presentar un matiz sobre la superación del dualismo, entre lo privado y lo público. Si bien esta escisión es superada desde la experiencia cotidiana y personal, es importante encontrar un punto de equilibrio que, conservando la sacralidad de lo íntimo, subsane la fractura existente, a través del establecimiento de relaciones significativas entre ambos espacios, sin perder su carácter propio. Ante una ruptura en la que el ser humano parece verse obligado a responder a diversos frentes sin ninguna relación entre ellos, se da una irrupción de lo privado en lo público que supone una difusión de límites, suscitando así un espacio distinto - político-religioso - en el que surgen aspectos y tareas en aras de la unión entre la vida interior y la vida social.

De esta forma, una comprensión del escenario colombiano posterior a los acuerdos de paz y en camino de superación del conflicto armado, encuentra en la co-implicación de lo religioso y lo político un referente fundamental donde encuentran sentido categorías como el bien común y la justicia, el perdón y la reconciliación, las comprensiones de la tierra y el arraigo, todas ellas en la lógica de afianzamiento identitario a nivel personal y comunitario.

En segundo lugar, respecto a la forma cómo esta comprensión llega a la escuela, se parte de la consideración del área de Educación Religiosa en el ámbito escolar contemporáneo, en una perspectiva que permita reconocer su importancia en relación con la formación humanística y la necesidad de una disciplina que la sustente. El punto de partida, remite a las transformaciones de la educación y la escuela en la sociedad contemporánea, resultado de la irrupción de la lógica del mercado como referente indispensable de la modernidad tardía de los países latinoamericanos; frente a un marcado énfasis por la competitividad, la formación para el trabajo y la capacitación para el desarrollo tecnológico, las omisiones más representativas están referidas a la concepción de la escuela como productora de cultura y las posibilidades políticas de cara a impulsar transformaciones sociales; aún menos importancia reciben apuestas como la atención a la dimensión trascendente del ser humano. 
Teniendo en cuenta lo anterior, y ante una educación que no ha privilegiado las humanidades, se plantea una recuperación de las mismas, no como un gueto con primacía y privilegio, sino en el marco del derecho a recibir una auténtica formación integral. De tal modo, la inclusión de elementos como lo público y la diversidad en la escuela, permiten ir más allá de una relación unidimensional con el conocimiento, que en ocasiones toma forma de reproducción del sistema ideológico dominante, para constituirse en una afirmación del ser humano en el mundo, donde le corresponde un ejercicio de afrontamiento y superación de condiciones de vulnerabilidad y exclusión; la realidad escolar de las Instituciones Educativas Distritales no es ajena a estas dinámicas.

Las implicaciones de lo expuesto, suponen el reconocimiento de ciertos vacíos epistemológicos en el ámbito escolar, que pueden estar contribuyendo a diluir su función de construir intersubjetivamente una cosmovisión que permita a las personas situarse en el mundo, en sus contextos particulares, y realizar diversas lecturas e interpretaciones del mismo. Así, entra en escena la Educación Religiosa en su proceso de configuración como saber escolar, atravesado por el cuestionamiento sobre su pertinencia académica y por el debate sobre su inclusión en la educación formal escolar que comporta intereses políticos.

En esa perspectiva, una consideración actual de la educación en su conjunto, incluye el abordaje de los distintos lugares y lenguajes de producción de conocimiento y desarrollo de las potencialidades humanas, entre las que se cuentan las preguntas existenciales del ser humano -el origen, la muerte, el sufrimiento, el destino- que constituyen una expresión de su dimensión trascendente. En este sentido, la cuestión epistemológica de la Educación Religiosa queda inicialmente remitida al problema del sentido de la vida y el alcance real que tiene la persona de conocer y aproximarse a este cuestionamiento; más aún, a la forma cómo un área de conocimiento puede ayudar en esa tarea, a través de un lenguaje que la haga posible en el ambiente escolar y en diálogo con otros saberes.

La opción termina decantándose por las denominadas Ciencias de la Religión, ante la necesidad de un saber disciplinar que estudiando lo religioso en su especificidad, al tiempo pueda entrar en contacto con otros saberes y con el mundo de las disciplinas, a partir de aquello que da unidad al conocimiento humano: la persona, productora de sentido para el mismo. La identificación de este referente, supone para la Educación Religiosa, la posibilidad de abarcar la trascendencia, a través de las elaboraciones culturales y de los modos de ser y desempeñarse de las personas, remitiéndolas a los cuestionamientos existenciales y a la búsqueda de sentido. Desde las Ciencias de la Religión, la Educación Religiosa como saber escolar adquiere un necesario sustento en la articulación con el currículo escolar con miras en el objetivo de educar en la apertura al mundo como presupuesto fundamental de la humanización. De tal modo, aspectos como el uso de la libertad, la participación activa de la sociedad, el respeto por 
los derechos humanos, y la formación de una ciudadanía consciente de su responsabilidad hacia el futuro, hacen parte de los elementos que constituyen el horizonte que comparte la Educación Religiosa con las humanidades. Bajo ese horizonte, y superada la visión de una confesionalidad con pretensiones de uniformidad, se asume para el contexto distrital, no la supresión de lo religioso en la escuela sino el reconocimiento de su carácter público, esto es, coadyuvando a la formación en la libertad y a la valoración de la diversidad.

En ese sentido, la pertinencia del área, en relación con la necesidad de reconstrucción del tejido social, radica en su contribución a la tarea de humanización, a través de la inclusión de elementos que posibilitan la construcción de sentido: prácticas de memoria histórica encaminadas a la resiliencia. Se ha planteado entonces, un saber escolar encaminado a la construcción de sujetos históricos que reconocen sus realidades contextuales y su realidad personal, en una dinámica de desarrollo de sus potencialidades como expresión del sentido de su vida y de su papel en la historia.

\section{Decolonización del ser: la apuesta por la trascendencia}

El replanteamiento de las concepciones entre los saberes y las disciplinas, y las relaciones entre ellas, tiene repercusiones directas en la concepción de persona y la forma en que esta se presenta ante el mundo desarrollando sus potencialidades; esto supone la generación de una conciencia crítica que a la larga constituya una conciencia liberadora. En la esfera del ser, la experiencia pedagógica apela a la dimensión trascendente del ser humano como mecanismo inmerso en la realidad que permite confrontarla, emergiendo dinámicas ligadas al reconocimiento del otro en el plano político.

La apuesta está dada por el reconocimiento de la dimensión trascendente del ser humano en su capacidad de hacer frente a la realidad en la que se halla inmerso; para ello, se parte de la pluridimensionalidad de la persona y su necesidad de asumir una postura de acuerdo con el contexto en el que se desenvuelve. Ahora bien, la situación actual remite necesariamente a fenómenos como la globalización y la sociedad de consumo que modifican las prácticas culturales, afectando a los niños y jóvenes en el campo de las subjetividades, las cosmovisiones y los estilos de vida. De hecho, entre los elementos identificados en las experiencias de los docentes en relación con los jóvenes de Instituciones Educativas Distritales, está la percepción de una carencia de sentido en una dinámica de desesperanza; esto se expresa de diversas maneras: desde el aislamiento -manifestado tanto en la separación de un grupo de referencia como en la apatía ante diversas opciones y alternativas-, hasta la búsqueda afectiva en relaciones de amistad y creación de intimidad, en superación de un panorama de superficialidad, consumo y fugacidad (VIDES SAN JUAN, 2016). Si bien algunos de 
los comportamientos corresponden a las características etáreas de la población, también son reflejo de aspiraciones más profundas hacia el compromiso, destinos compartidos o sentimientos de fraternidad.

En este escenario, y como derivación de la reducción de la religión al ámbito de lo privado, la trascendencia ha sido reducida a un cerco intimista quedando separada de las intencionalidades e intereses del marco social, de donde se deriva el reto de recuperar una comprensión holística de lo social, donde las interconexiones permitan una reconfiguración de sentidos en las redes, imaginarios y estilos de vida de las personas concretas. La comprensión de lo religioso como alternativa para la superación de visiones reduccionistas y ante la necesidad de elaborar continua y significativamente un sentido para la existencia, remite necesariamente a la dimensión trascendente de la persona. Dos elementos permiten hacer esa correlación: por una parte, la consideración de la contingencia del mundo y del ser humano, desde la cual es posible comprender la trascendencia como alternativa de crecimiento personal, en un horizonte no intimista sino como postura de afrontamiento ante realidades exigentes y a la comprensión fragmentada de las mismas; por otra parte, partiendo del contraste entre la idea de lo efímero, y la de permanencia en el tiempo, el planteamiento de la brevedad de la existencia personal expresada en la mortalidad encuentra esperanza en la cultura como mecanismo de perdurabilidad, basado en la concepción de que las creaciones y elaboraciones personales y sociales tienen un significado perenne trascendiendo más allá de la muerte. Teniendo esto en cuenta, resulta fundamental la función de lo religioso en el campo de la producción cultural como mediación de sentido existencial.

De esta forma, el planteamiento de lo religioso como referente de comprensión de la realidad, admite la afirmación de la vida cotidiana como el espacio de desenvolvimiento de la dimensión trascendente, desde la afectación de la totalidad de la experiencia humana, a raíz de los dinamismos sociales y culturales contemporáneos. Una auténtica reconstrucción del tejido social debe ser vinculante de los referentes culturales como expresión del sentido generado a nivel personal y comunitario, y manifestado, en el caso latinoamericano, en elementos como la importancia simbólica de la tierra, y la autenticidad y solidez de las relaciones interpersonales.

La dimensión trascendente resulta ser así, un factor potenciador del ámbito cultural y político. Retomando la perspectiva del contexto colombiano, donde la violencia se ha hecho manifiesta en el conflicto armado, pero también en otras situaciones cotidianas, es evidente la necesidad de romper con esas dinámicas instaladas en la cultura; para ello, hay que volver al problema que se planteó inicialmente: el reconocimiento del otro, esta vez retomado en tres perspectivas del ser, que hacen explícitos algunos elementos del ámbito político. 
En primer lugar, una de las tareas fundamentales en el escenario colombiano, es la recuperación de la confianza. De hecho, el debilitamiento de las relaciones de los individuos entre sí, y de estos con las instituciones, hacen que el fomento de una nueva credibilidad sea primordial. Para ello es necesario recordar que, por un lado, se da la confianza entre pares, lógica en la cual se parte de la convicción de que el otro no atentará contra mí y propugnará por el bienestar comunitario; y, por otro lado, aparece la relación de los individuos con las instituciones, su papel en la sociedad y el grado de legitimidad que se les concede. En correspondencia, en un marco de violencia, en primer lugar, se hace imperativo recuperar los niveles de reconocimiento del otro como igual y de la acción colectiva como movilización posibilitadora de una transformación social, y, en segundo lugar, se evidencia la necesidad de reformas que permitan redefinir la relación de los ciudadanos con las instituciones, en el sentido de reconquistar la confianza en un estado que en principio permitió la vulneración de derechos. De esta manera, el reconocimiento pleno del otro, implica una postura de confianza indispensable para los procesos de reconstrucción de la unidad nacional; hay allí, cierta tensión entre lo personal y lo estructural, que puede expresarse desde la importancia de convencer a las personas, es decir un trabajo desde las convicciones y el sentimiento moral, pero, asimismo, la exigencia de estructuras que garanticen la permanencia de las políticas de reconstrucción del tejido social.

En segundo lugar, alimentar la esperanza basada en el anhelo de justicia, implica, especialmente en el escenario colombiano, una consideración inicial de la relación entre justicia y paz, en un marco democrático. De hecho, la concepción de una democracia auténtica donde existan mecanismos legítimos de inclusión de las minorías y la diversidad, desafía toda forma de exclusión en cuanto invisibilización y negación del otro; esto permite vincular íntimamente la justicia con la dignidad humana en el reconocimiento de que un escenario de violencia transforma la vida de las personas, en una forma imposible de reparar plenamente, y más aún, exige a la sociedad hacer posible la escucha de la voz de todos, factor indispensable en una democracia real. En esa línea, adquieren relevancia los espacios de visibilización social y reivindicación de la dignidad, en un proceso que vincula tanto la ampliación del concepto de Verdad, -implicando una aceptación de lo diverso que puede expresarse en múltiples verdades, asumiendo una comprensión enriquecida del legado histórico-, como la consideración de los actores participantes en un conflicto, -ya no sólo víctimas, desplazados, campesinos, sino también, guerrilleros, paramilitares, ejército- más que desde el prejuicio y el estigma social, desde su condición personal: individuos con rostro, historia, intereses, motivos, y más aún insertos en un contexto que está cambiando y al que necesitan ajustarse y dar una respuesta.

En tercer lugar, apelar a las experiencias de dolor y sufrimiento, como generadoras de sentido en torno a lo auténticamente humano, en cuanto reivindica 
el papel fundamental y primero de la persona concreta. El proceso de asumir las propias experiencias e involucrarlas en la fundamentación de certidumbres morales y de posicionamiento frente a la realidad social y política, posibilita la afirmación del ser personal en cuanto ser abierto en alteridad y la necesaria transición del dolet ergo sum (me duele, luego existo), -planteado por Kierkegaard en el intento de superación del cogito ergo sum cartesiano-, al doles ergo sum (me dueles, luego existo); esta propuesta de Carlos Díaz (2006), implica la humanidad en plenitud en cuanto supone el espacio relacional donde el ser personal se siente afectado, no por la humanidad que se convierte aquí en un concepto abstracto, sino por el rostro concreto de quien sufre. De lo anterior se derivan, entre otros planteamientos: primero, la prioridad de quien sufre, expresada en la máxima: "cuando alguien que no eres tú, llora, tiene derecho sobre ti, por eso cuidar a un ser humano que sufre es lo más urgente" (DIAZ, 2006, p. 85); segundo, la exigencia de un mayor compromiso que supere el planteamiento de estar con el otro, por el de estar para el otro, es decir, más allá de discursos y posiciones radicales, lo que se demanda es cercanía y diligencia, lo cual hace a la persona vulnerable e incapaz de apartar la mirada ante el dolor del otro. Y finalmente, una nueva comprensión del otro-enemigo, más aún en las circunstancias de conflicto; dice Díaz:

nadie tiene derecho de llamar malo a ningún ser humano. Sólo tenemos derecho a distinguir lo bueno de lo malo, pero no al bueno del malo. En cuanto borremos al malo del léxico de nuestra conciencia nos veremos libres del fantasma del enemigo (DIAZ, 2006, p. 88).

\section{Decolonización de las prácticas culturales: la cultura como puerta de entrada a lo existencial}

Como ya se ha esbozado, la decolonización del saber, al comportar implicaciones en el campo de las subjetividades, también reelabora comprensiones a nivel socio-cultural sobre prácticas que se asumen como deseables o legítimas; esto supone una atención a los movimientos y manifestaciones sociales cuya conciencia crítica es expresión de las múltiples formas en que lo subjetivo va constituyendo estructuras colectivas en un afán de reivindicación. En relación con la experiencia de comprensión del conflicto armado y su necesidad de superación, en el marco del área de Educación Religiosa, esto implica partir del reconocimiento de lo religioso como hecho cultural, de cuya condición se deriva una vinculación al ámbito político; así mismo, se plantean lineamientos respecto a las implicaciones escolares, en términos de núcleos de formación que relacionan lo cultural con lo existencial.

Entender lo religioso como expresión de la condición cultural inherente al ser humano, supone necesariamente la comprensión de que la cultura es resultado de una actividad de significación que la persona hace de su entorno; así, la pertenencia del hecho religioso a este sistema de significación lo constituye 
tanto en hecho cultural, por ser expresión de una manera específica de pensar y de vivir del ser humano, como en hecho religioso propiamente, por constituir el lugar de objetivación visible de la relación entre los seres humanos y la trascendencia. Esta concepción de lo religioso, -a partir de los sistemas de símbolos y significados que constituyen y definen sus manifestaciones-, ofrece una mirada encaminada a comprender el sentido del entorno como totalidad, en relación con normas y valores que establecen un universo simbólico que supera lo cotidiano; con ese referente, es posible afirmar que la Educación Religiosa mediante una relectura de lo cotidiano, coadyuva a la formación de un individuo que comprende su contexto sociocultural, político, económico y religioso.

De esta manera, se favorece un relacionamiento con el mundo como descubrimiento del otro en sus realidades y lenguajes; y en esa medida, se contribuye a la tolerancia, a no establecer juicios desde los propios referentes sino en reconocimiento de nuevos sentidos y valores en el marco de la diversidad. En el mismo sentido, la opción por el conocimiento religioso implica necesariamente una comprensión de la vivencia de la propia cultura, la convivencia con distintas tradiciones religiosas y el respeto a las diversas formas de expresión cultural. De hecho, la promoción de lo religioso como referente cultural pasa, por una parte, por el reconocimiento que la pertenencia a una comunidad supone necesariamente asumir una responsabilidad en ella, y por otra parte, por la comprensión de la ciudadanía no desde la pertenencia a una nación o país, sino a la especie humana, es decir, desde la condición de persona; ambos aspectos poseen implicaciones políticas en el campo de la convivencia, las relaciones interpersonales y los vínculos de las personas con las instituciones.

Con base en lo anterior, la relación de la Educación Religiosa con algunos elementos de la formación política, se da en la finalidad de formación de ciudadanos, que, en reconocimiento de la dignidad de la persona, se comprometan con la realidad social en que viven y fomenten actitudes de aceptación hacia la diferencia. A esto contribuye el desarrollo de habilidades de carácter relacional, encaminadas al aprender a vivir juntos, a trabajar en forma cooperativa, a valorar y promover la democracia, entre otros aspectos, que, en últimas, dan cuenta de una experiencia de lo religioso que se traduce en una capacidad de humanización manifestada en relaciones humanas de calidad.

Ahora bien, si el papel de lo religioso resulta fundamental en la afirmación de la identidad de las personas y comunidades, desde sus referentes históricos y culturales, y de ello se derivan elementos relevantes para la formación política, se hace necesario comprender como esa vinculación cultural puede encausar posibilidades pedagógicas. Con ese objetivo, se plantean algunas apuestas coherentes con lo ya expuesto sobre las relaciones entre lo religioso y lo político, a modo de lineamientos que han ido permitiendo elaboraciones, de acuerdo con las realidades y contextos específicos de las Instituciones Educativas Distritales. 
El punto de partida es el ser humano, no en abstracto sino desde las personas concretas que necesitan herramientas para responder los cuestionamientos sobre su existencia y su papel en el mundo; una educación integral auténtica implica coadyuvar a la elaboración personal de una cosmovisión como referente para enfrentar las razones que afectan los modos de vivir y las opciones que se asumen en uso de la libertad. Esto supone para la Educación Religiosa un carácter, no sólo de conocimiento y análisis de la realidad, sino de proposición y producción, en coherencia con las implicaciones de la dimensión trascendente. De hecho, el estudio de lo religioso abre paso a la percepción de la propia contingencia y sus manifestaciones en la historia de la humanidad aludiendo a temas como la identidad, la alteridad, la autonomía, que resultan como justificación de dinámicas de inclusión y exclusión.

Desde esta perspectiva, las disciplinas que se identifican bajo el conjunto de las Ciencias de la Religión, comparten un potencial liberador, en cuanto aportan en la construcción de una visión de lo religioso en la historia y el presente, como referente de realidad y relectura de lo cotidiano. Esta ubicación posibilita aproximaciones con otras áreas, que, comparten dinamismos que no parecen responder del todo a la lógica de los saberes escolares determinados por un saber disciplinar de corte puramente académico, sino que incluyen un elemento práctico vivencial en los sentidos tanto social como trascendente. Dicho esto, se plantea como referente de elaboración pedagógica, lo religioso en sí mismo, desde las consideraciones que lo remiten a las articulaciones socio-culturales que expresan la dimensión trascendente del ser humano, y que admiten como válida y necesaria una co-implicación con lo político. En este sentido, las religiones son susceptibles de estudio en cuanto manifestaciones de lo religioso, esto es, como producción cultural y expresión de la libertad de las personas y comunidades en su tendencia a realizarse y a alcanzar la plenitud. Esta comprensión implica para el ámbito educativo distrital una transición de la percepción generalizada de que lo religioso no incumbe a la escuela pública, hacia el reconocimiento de que el deber de la escuela es asumirlo como parte de la construcción de la cosmovisión que realizan los niños y jóvenes; la Educación Religiosa se configura, así como un lugar de interpelación de la realidad a partir de su problematización.

De tal modo, se derivan dos núcleos de formación a los cuales tendría que atender en sus posibilidades escolares: el Histórico-Cultural, que remite a la pertenencia de las religiones al campo de las expresiones culturales, es decir, el descubrimiento del legado de lo religioso en la sociedad, y el Existencial, que da cuenta de los cuestionamientos de la dimensión trascendente como parte del proceso de formación integral del ser humano. Cabe anotar el carácter complementario de esta división, en reconocimiento que lo cultural sólo encuentra sentido como manifestación de la libertad humana en su aspiración a la plenitud. 
El primer núcleo, parte de la historia y del papel de la tradición religiosa en la estructuración y mantenimiento de las diferentes culturas; apela a un acercamiento a los códigos culturales como estructuras de significación que reflejan la relación del ser humano con lo que considera sagrado. De tal manera, reconoce las manifestaciones religiosas como patrimonio de la humanidad, en cuanto hacen parte de los contenidos elaborados históricamente por las comunidades y que constituyen su fundamento cultural; en esa línea, su conocimiento y apropiación escolar supera el enfoque puramente patrimonial para ser una contribución a la comprensión del mundo contemporáneo y el posicionamiento del estudiante en su realidad.

Por su parte, el núcleo Existencial se halla más cercano a cuestiones que tradicionalmente se vinculan con el pensamiento filosófico. Implica para la Educación Religiosa, la inclusión de las preguntas de la persona por su propio ser, su origen y su destino, que resultan vinculadas tanto a formas de organización social, como a expresiones culturales, siendo representación de las maneras como los seres humanos asumen el enfrentamiento con el misterio. Esto supone para los estudiantes su ubicación en un proceso de búsqueda de sentido, cercano a la afirmación personal, a través de la consideración de las propias preocupaciones y esperanzas; en esta perspectiva, lo religioso es leído como posibilidad de respuesta a inquietudes que afectan al ser humano como especie, pero asimismo a la persona concreta. Se asumen en este núcleo alternativas como el trabajo a partir del mito en su capacidad de respuesta al misterio, iniciativas de autoconocimiento y fomento de posturas frente a problemas universales como la injusticia, la ignorancia y el sufrimiento.

A través de ambos núcleos se posibilita, por una parte, la comprensión de la realidad, con su toma de posición en ella, esto es, la relación con la cotidianidad que supone unas implicaciones sociales; y, por otra parte, constituir un aporte a la paz y a la convivencia desde la comprensión del otro en su ser y actuar, $y$, a la reconciliación en reconocimiento de que el otro necesita un espacio en el mundo. El cumplimiento de estos propósitos conlleva una adecuada escogencia y planteamiento, de ejes problémicos relevantes desde la lectura e interpretación de la realidad; elementos como la resiliencia, la memoria y la reconciliación, cuya necesidad e importancia es imperativa en contextos de conflicto armado, constituyen una respuesta al cuestionamiento por la unión de los núcleos Histórico-cultural y Existencial, al conjugar en la práctica elementos que conjugan la íntima conexión entre las expresiones culturales y el ámbito trascendente, que se da en la posibilidad de dotar de sentido la vida y los acontecimientos. A un abordaje de estas perspectivas, se dedica el último apartado.

\section{Decolonización del currículo: caminos hacia la resiliencia}

La implementación en la escuela de los núcleos planteados, y particularmente del núcleo existencial, se ha constituido en un reto alimentado por las 
necesidades conceptuales del escenario colombiano. A continuación, se presentan algunas posibilidades que se han ido descubriendo, como parte de los aprendizajes del proceso en las prácticas concretas y que han ido tomando forma en la búsqueda de ejes orientadores que admitan la doble relación con lo político y lo trascendente, de cara a las convicciones de las personas, su cosmovisión y su desempeño en el mundo.

Ya Argüello Parra (2015), planteó tres tendencias privilegiadas en las pedagogías decoloniales: el fomento y preservación cultural, donde se destaca la educación rural e indígena y la ancestralidad de la mano de los patrimonios orales intangibles; el análisis y transformación sociopolítica, que permite abordar la paz, el posconflicto, el desplazamiento forzado, así como temas de género y de violencia; y las tendencias ecosóficas, más ligadas a las economías y desarrollos alternativos y la biodiversidad. En el escenario de la Educación Religiosa ligada a la formación política, esa apertura de perspectivas para la interpretación de la realidad, ha permitido incursionar en los ejes de Paz, Reconciliación y Memoria, constituyendo un ámbito de unión entre lo histórico-cultural y lo existencial, que privilegia los acontecimientos pasados y presentes como forma de afirmación identitaria y proyecta escenarios futuros en conexión con la transformación y reconstrucción del tejido social; se asumen estos tres ejes cuya inclusión en el currículo, consolida los derroteros hacia la Resiliencia, categoría transversal fundamental.

La opción por privilegiar la categoría Resiliencia se deriva de las circunstancias particulares de una sociedad, enfrentada a sanar heridas, recuperar la confianza y adaptarse a nuevas circunstancias después de décadas de conflicto armado. De hecho, el término tiene su origen en la física para describir la resistencia de los metales al ser sometidos a altas presiones recobrando su forma original, y su adaptación a las ciencias sociales, implicó reconocer las capacidades y habilidades de ciertas personas para sobreponerse a situaciones de riesgo y adversidades, desarrollando una situación de bienestar en distintos niveles. La promoción de la resiliencia se encamina a lograr el desarrollo pleno de la persona en un ambiente facilitador de forma que al centrarnos en las características y potencialidades de cada uno éste pueda enfrentar situaciones adversas y salir fortalecido; esta capacidad de afrontamiento supone una transformación de la persona en desarrollo de habilidades que le permiten la superación de experiencias difíciles, y adquiere importancia en fenómenos sociales donde la supervivencia y el desarraigo marcan derroteros que afectan de forma directa el desarrollo de las personas.

Una iniciativa escolar encaminada al desarrollo de la resiliencia se debe centrar en el fomento de las habilidades que permiten a la persona superar una situación difícil y que en el caso preventivo se constituye en escudos protectores en contra de las fuerzas de violencia. En esta perspectiva, resulta fundamental el empoderamiento de la persona en el sentido de favorecer la confianza en sí mismo y en las habilidades que tiene para superar una situación desfavorable. Para 
ello, de acuerdo con Munist et al. (1998), se deben privilegiar: primero, la competencia social, dónde el contacto con otros seres humanos permite generar más respuestas positivas; segundo, la resolución de problemas donde hay un punto importante para el fomento de la creatividad y soluciones alternativas; tercero, la autonomía, entendida desde la propia identidad y la habilidad para actuar de forma independiente ejerciendo control sobre algunos factores personales y el entorno; y, cuarto, el sentido de propósito y de futuro, desde la formulación de expectativas saludables y la motivación para su consecución. Este último adquiere importancia para el caso educativo, para el fortalecimiento de las aspiraciones educativas y el anhelo por un futuro mejor, en términos posibles y alcanzables.

Ahora bien, el fomento de la resiliencia supone una interacción entre la persona y su contexto, de donde el referente contextual, mediado por los sentidos y significados que circulan, resulta fundamental. De hecho, las características sociales y culturales son fundamentales para reconocer que no existen los niños y jóvenes en abstracto, y que en ese sentido, asumir con respeto las características particulares de la cotidianidad de los estudiantes, contribuye a fortalecer su identidad personal y comunitaria; del mismo modo, asignarles un rol protagónico, a través de procesos de debate para discernir las acciones posibles más pertinentes y eficaces, fortalece su destreza para resolver problemas y su confianza en las propias capacidades personales y comunitarias. Dicho esto, la importancia de la dimensión cultural está dada por la pertinencia pedagógica de los esfuerzos que la escuela o los programas educativos desarrollen.

Un último aspecto respecto a la Resiliencia, está en su íntima relación con la trascendencia, en los términos ya expuestos: en cuanto permite un abordaje y confrontación con la realidad y remite a las preguntas existenciales del ser humano; en el caso de los adolescentes en proceso de comprensión de una sociedad marcada por la violencia, esto implica un retorno a su propia historia desde la infancia, así como a las historias familiares, como fuente de sentido de cara a la toma decisiones óptimas ligada al deseo de desarrollarse plenamente. Así, la coincidencia entre Resiliencia y trascendencia, se da en sus relaciones con el ámbito cultural y en la búsqueda de significado y sentido para la vida; a partir de ahí, esa conexión constituye una herramienta fundamental de adaptación y superación en situaciones exclusión, violencia y desarraigo. Cabe recordar que estas dinámicas, siendo profundamente personales, al tiempo multiplican su eficacia cuando se alimentan y apoyan en una comunidad de referencia.

Finalmente, un breve abordaje sobre la Paz, la Reconciliación y la Memoria, categorías constituyentes del ámbito pedagógico de Resiliencia, donde se pretende fomentar la superación de situaciones adversidad, específicamente el caso de la violencia física y política. Inicialmente, la inclusión de la Paz al currículo supone para el sistema educativo, una regulación de las expectativas sociales, superando la concepción mesiánica que quita responsabilidades sobre 
las transformaciones a otros sectores sociales, y comprender la multidimensionalidad de la noción de Paz, para incluir en su estudio el establecimiento de ciertas condiciones de posibilidad. En este sentido, la ruta de trabajo pasa por el análisis histórico, la construcción de la memoria y el conocimiento sobre las dinámicas sociales y políticas, con miras en la consolidación de una cultura que privilegie el respeto de los derechos humanos y la preservación de la vida como ejes primordiales. Lo anterior implica superar el riesgo del activismo por la paz donde la educación puede ser reducida a la reproducción de una paz hegemónica fragmentada y coyuntural, evitando poner sobre la mesa temas y decisiones paradigmáticas como la distribución de la tierra, los conflictos de género en medio del conflicto y las implicaciones de reparación hacia las víctimas.

Dicho lo anterior, lo que entra en juego es la pregunta por el tipo de educación que requiere Colombia después del conflicto armado, y en ello, la forma como la escuela está contribuyendo a la comprensión de las causas de la violencia, a la empatía con los distintos actores involucrados, y el acompañamiento a los territorios, de forma que las medidas superen la simple declaración de buenas intenciones. La perspectiva que se plantea busca encaminar un proceso contextualizado, atravesado por prácticas que incluyen la revisión de procesos de transición de la guerra a la paz, cartografías del conflicto, análisis de los impactos de la violencia, establecimiento de relaciones con otras dinámicas como la corrupción y el narcotráfico, entre otros; sin embargo, la distinción entre esta educación sobre la Paz, -basada en fundamentos, dimensiones y relaciones conceptuales-, y la educación para la Paz, se da por la conexión con las dinámicas personales, en términos de actitudes y prácticas cotidianas que involucran aspectos afectivos, éticos, estéticos, y por supuesto, la relación con las preguntas existenciales del ser humano sobre la muerte, el dolor y el propósito de la vida, que no son ajenas a un escenario de violencia.

Esta complementariedad entre la educación sobre la Paz y la educación para la Paz, permite la generación de conciencia crítica y habilidades personales, entre las que se cuenta el acercamiento al otro, incluso cuando ha estado involucrado en situaciones de vulneración de mis derechos; en otras palabras, de lo que se trata, es de la inclusión de la Reconciliación en una perspectiva curricular. Cabe anotar que la complejidad del concepto, no ha permitido que sea asumido en sus múltiples aristas en la escuela, obviando las diversas posibilidades de desarrollo pedagógico; sin embargo, muchas de las comprensiones sobre las prácticas de relacionamiento social entre los actores de la comunidad educativa, pueden encontrar una reformulación enriquecedora a partir de este principio. De hecho, siguiendo a Téllez Salazar (2016), las iniciativas y orientaciones tendientes al manejo de conflictos escolares comportan un enfoque más normativo que formativo, creando una cultura resistente al abordaje de los problemas reales, que constituyen necesidades sentidas por parte de la sociedad representada en 
los estudiantes inmersos en contextos de vulnerabilidad; el enfoque normativo predominante es no sólo innecesario sino en ocasiones contraproducente con la función pedagógica de la escuela en el campo de la construcción social. De ahí la necesidad de fomentar una cultura escolar alternativa fundada en la autonomía, en formas distintas de resolver el conflicto, superando el paradigma sancionatorio y excluyente, y contribuyendo a procesos de reconciliación auténtica.

Un reconocimiento del conflicto en general, pero también de los propios conflictos como negación del otro, es la puerta de entrada a una cultura escolar que supere las exigencias legales en esa materia. Procesos educativos significativos para el fomento de una cultura de reconciliación, por una parte, se alimentan de una sólida formación política en términos de conocimiento, aceptación y valoración del otro en su diferencia; y por otra parte, suponen una concepción pedagógica plural creativa, que en lo estructural genere prácticas participativas, dialógicas y críticas; en lo ético y cultural, asuman componentes afectivos y emocionales como la empatía, la ética del cuidado, y las relaciones entre lo subjetivo y lo intersubjetivo; y desde el posconflicto, establezca una comprensión clara de la cultura de la legalidad, la verdad y la justicia desde la formación de la conciencia moral (ARIAS CAMPOS, 2016).

Finalmente, la inclusión al currículo de la Memoria histórica parte del reconocimiento que su consolidación es un logro social, junto al esclarecimiento histórico, porque permite dar sentido a las experiencias a través de las narrativas heterogéneas que dan cuenta de una construcción intersubjetiva de la realidad social, más aún, en contextos de violencia masiva. Dicho esto, su abordaje escolar no es el de una experiencia del pasado, sino una expresión de rebeldía frente a la violencia y la impunidad, que tiene repercusiones en el presente, y permite proyectar rutas de reconstrucción social hacia el futuro. En ello resulta indispensable intentar un equilibrio entre la diversidad y la identidad, de forma que el enriquecimiento de las relaciones interpersonales que se da en el proceso de reconstrucción de la propia historia, se convierta en mecanismo que potencie en los estudiantes la conciencia crítica sobre la propia realidad, el empoderamiento y la movilización comunitaria de talante transformador.

En este sentido, prácticas como la elaboración de árboles genealógicos y de autobiografías, se asumen en una perspectiva tanto personal, en reconocimiento del talante individual e íntimamente personal de la memoria, como inclusiva, en cuanto posibilita la participación de todos. De hecho, la capacidad generadora de sentido que tiene la subjetividad al momento de expresar la experiencia pasada, su presentificación y las posibilidades transformadoras en el futuro, se multiplica en la interacción con otros que a su vez están en el proceso de descubrimiento de múltiples significados. Esta remisión a la diversidad en las experiencias y en las prácticas culturales, implica en sí misma una apuesta por fomentar conciencia desde la diferencia y la valoración del otro, así como desde el fortalecimiento los vínculos dialógicos. 
De esta forma, la Paz como camino y meta, la Reconciliación como mecanismo de desarticulación de las violencias culturales, y la recuperación de las Memorias individuales y colectivas, constituyen un ámbito favorecedor de la Resiliencia, en cuanto contribuyen a dotar de sentido la realidad social mediante la construcción intersubjetiva de una cosmovisión encaminada a la reconstrucción del tejido social. Se considera allí una posible respuesta a la pregunta por la unión en la práctica de los núcleos histórico-cultural y existencial de la concepción de Educación Religiosa ligada a la formación política.

\section{Consideraciones finales}

Se ha presentado, una sistematización en clave decolonial, de las reflexiones inspiradas en una experiencia pedagógica vinculante de la Educación Religiosa escolar a la formación política, en el contexto del posconflicto en Colombia. Las exigencias de este contexto han implicado: en el campo del saber, la reconexión de lo privado y lo público, reflejado en la complicación de lo político y lo religioso subvirtiendo la separación moderna en ese sentido; en el campo del ser, la promoción del respeto a la identidad y a la diversidad se plantea en perspectiva de formación de personas que en uso de su libertad comprendan y respeten los derechos de todos; en el campo de las prácticas culturales, se descubre la forma en que la cultura, en cuanto red de significados, abre paso a la reflexión existencial en reconocimiento de la íntima unión entre expresiones culturales y trascendencia; en esa línea, la unión de los núcleos histórico-cultural y existencial, permite la construcción intersubjetiva de una cosmovisión vinculante de elementos políticos; finalmente, el proceso lleva al descubrimiento de la Resiliencia como referente cuya concreción curricular puede permitir la consolidación de derroteros encaminados a la necesaria reconstrucción del tejido social.

Ahora, si bien emerge una tensión entre los referentes teóricos y el nivel de las realidades ya que la construcción social parte de intuiciones no siempre correspondientes con supuestos abstractos, las prácticas escolares en las que se incluyen la reflexión sobre la diferencia y la valoración de quienes comprenden el mundo de forma alternativa, se constituyen en posibilidad del derecho de expresión de la diversidad como ejercicio de la ciudadanía. Teniendo en cuenta lo anterior, desde la perspectiva decolonial le educación supera las expectativas académicas para comprometerse con la acción y el compromiso de transformación social que se traduce en una ruptura de los mecanismos que mantienen la negación del otro; aparece allí un marco de comprensión que exige la renovación de las dinámicas educativas, especialmente en los ámbitos formales generalmente mediados por las estructuras rígidas de la escuela tradicional.

Teniendo en cuenta lo anterior, las contribuciones de la educación a la configuración de una sociedad resiliente, se plantean en la comprensión alter- 
nativa de la Paz, superando los discursos hegemónicos que pueden terminar fragmentándola y obviando algunas de sus dimensiones más significativas. En una coyuntura como la colombiana, la promoción de la Resiliencia debe ser una prioridad en el contexto educativo, especialmente desde la creación de ambientes que permitan reconstruir sobre circunstancias con factores adversos. Para ello, se esbozan finalmente dos grandes retos.

Primero, el fortalecimiento de un enfoque territorial que acepta la conexión de la memoria y la identidad con los lugares, en una perspectiva donde el arraigo ligado a la experiencia de construcción personal, es generador de sentido. Un acercamiento al territorio en sus distintas dimensiones, permite entre otros aspectos: la integración entre el mundo urbano y el mundo rural en un sentido de aceptación y acogida de lo distinto; una apropiación del pasado, más allá de los documentos y apelando a la dignidad de los actores del conflicto; y, el descubrimiento de lógicas discursivas y lugares de enunciación alternativos entre los que se cuentan las estéticas de la memoria: cantos, música, imágenes, los cuerpos. Segundo, generar caminos que manifiesten la apuesta por una comprensión desde lo afectivo, esto es, el reconocimiento de la empatía como presupuesto para una interpelación de la realidad que en buena parte no es racional sino emocional. Esto supone que el conocimiento de la historia del país y de la propia historia, es una mediación para reconocer el propio lugar de desempeño y desde ahí: reconocer el dolor del otro, no sólo para presentificar la tragedia pasada sino para vislumbrar caminos de esperanza; recuperar la confianza como premisa necesaria para el abordaje de narrativas de memoria; y, construir testimonio, desde la pluralidad reconociendo que el conflicto armado nos afecta a todos mas no a todos de la misma manera.

Recebido em: 02/04/2019

Revisado pelo autor em: 19/04/2019 Aceito para publicação em: 08/05/2019

\section{Nota}

1 Doctor en Educación y Sociedad de la Universidad de La Salle (Bogotá). Magíster en Filosofía Latinoamericana de la Universidad Santo Tomás (Bogotá). Licenciado en Educación con especialidad en Estudios Religiosos de la Universidad de La Salle (Bogotá). E-mail: demmanuel01@gmail.com

\section{Referencias}

ARGÜELLO PARRA, Andrés. Pedagogía decolonial: Trazos para la construcción de un paradigma-otro desde la educación. Correo del Maestro, año 19, n. 226, p. 28-37, 2015. Recuperado de: https://www.correodelmaestro. com/publico/html5032015/capitulo4/Pedagogia_decolonial.html. Acceso en: 20 feb. 2019. 
ARIAS CAMPOS, Rosa. La educación para la paz: retos de las universidades en el posconflicto armado. Cuadernos de Seminario, n. 5, p. 13-26, 2015.

CELIS, Luis Eduardo. Política y violencia en Colombia. Seminario Virtual Caja de Herramientas, Bogotá, Colombia, n. 164, p. 1-8, 2009. Recuperado de: http://viva.org.co/cajavirtual/svc0164/articulo0002.pdf. Acceso en: 20 feb. 2019.

DÍAZ, Carlos. Por respeto al otro. Madrid: Dos Mundos, 2006.

DUCH, Lluís. Religión y Política. Barcelona: Fragmenta, 2014.

MUNIST, Mabel et al. Manual de identificación y promoción de la resiliencia en niños y adolescentes. Washington: Organización Panamericana de la Salud, 1998.

TÉLLEZ SALAZAR, Jairo. La reconciliación y el perdón en el sistema educativo colombiano. Cuadernos de Seminario, n. 5, p. 93-100, 2015.

VIDES SAN JUAN, David. La Educación Religiosa Escolar como espacio de formación política. Marco antropopedagógico desde las experiencias de docentes de Instituciones Educativas Distritales de Bogotá. 2006. Tesis (Doctorado en Educación y Sociedad) - Universidad de la Salle, Bogotá, 2016. 